\title{
ON THE SOLVABILITY OF SYSTEMS OF INCLUSIONS INVOLVING NONCOMPACT OPERATORS
}

\author{
P. NISTRI, V. V. OBUKHOVSKII, AND P. ZECCA
}

Abstract. We consider the solvability of a system

$$
\left\{\begin{array}{l}
y \in \bar{F}(x, y), \\
x \in \bar{G}(x, y)
\end{array}\right.
$$

of set-valued maps in two different cases. In the first one, the map $(x, y)-0$ $\bar{F}(x, y)$ is supposed to be closed graph with convex values and condensing in the second variable and $(x, y)-0 \bar{G}(x, y)$ is supposed to be a permissible map (i.e. composition of an upper semicontinuous map with acyclic values and a continuous, single-valued map), satisfying a condensivity condition in the first variable. In the second case $\bar{F}$ is as before with compact, not necessarily convex, values and $\bar{G}$ is an admissible map (i.e. it is composition of upper semicontinuous acyclic maps). In the latter case, in order to apply a fixed point theorem for admissible maps, we have to assume that the solution set $x \longrightarrow S(x)$ of the first equation is acyclic. Two examples of applications of the abstract results are given. The first is a control problem for a neutral functional differential equation on a finite time interval; the second one deals with a semilinear differential inclusion in a Banach space and sufficient conditions are given to show that it has periodic solutions of a prescribed period.

\section{INTRODUCTION}

This paper is devoted to the study of the solvability of a system of the form

$$
\left\{\begin{array}{l}
0 \in F(x, y), \\
0 \in G(x, y),
\end{array}\right.
$$

where $F$ and $G$ are maps given by

$$
F(x, y)=y-\bar{F}(x, y) \text { and } G(x, y)=x-\bar{G}(x, y)
$$

with $x \in X, y \in Y ; X, Y$ Banach spaces; $\bar{F}: \bar{U} \times \bar{V} \multimap Y, \bar{G}: \bar{U} \times \bar{V} \longrightarrow X$ and $U \subset X, V \subset Y$ are open sets with $0 \in U$.

In $\S 2$, first we give two results, Theorems 2.1 and 2.2 , concerning the case when the map $\bar{F}$ is convex-valued with closed graph and $\left(k, \psi_{Y}\right)$-condensing,

Received by the editors January 24, 1992.

1991 Mathematics Subject Classification. Primary 47H04, 34K35, 34C25.

Key words and phrases. Condensing multivalued map, degree theory, Borsuk-Ulam condition, control problem, periodic solution.

The first and third authors were supported in part by the research project M.U.R.S.T. (40\%) "Teoria del Controllo dei Sistemi Dinamici" and by a bilateral project C.N.R.; the second author was supported by C.N.R. 
$k \in[0,1)$, in the second variable with respect to a regular monotone nonsingular measure of noncompactness and $\bar{G}$ is a permissible map (i.e. it is the composition of an acyclic map and a continuous single-valued map) satisfying a certain condensivity condition in the first variable.

Moreover, in Theorem 2.3 we also give sufficient conditions for the existence of solutions of system $(0.1)$ when $\bar{F}$ is as before but it has arbitrary compact values and $\bar{G}$ is an admissible map (i.e. it is the composition of acyclic maps). In this case, we do not require, as in Theorems 2.1 and 2.2, any assumption on the topological degree of the vector field $F=I-\bar{F}$, but we have to assume that the solution set $S(x)$ is acyclic, in order to apply a fixed point theorem for admissible maps. Notice that this assumption arises naturally in applications.

In the case when $\bar{F}$ is an upper semicontinuous, compact map with convex, closed values, sufficient conditions for the solvability of system $(0.1)$ are given in [4]. The case when $\bar{F}$ is not convex-valued and the second equation is single-valued has also been treated in [5].

The approach we use in this paper follows closely that one of [4, 5 and 10]. That is, we solve the first equation with respect to $y$ treating the variable $x$ as a parameter and then we introduce the corresponding solution set $S(x)$ in the second equation. Obviously, the fixed points of the composite function $\bar{G}(x, S(x))$ are the solution of system $(0.1)$.

In order to follow such a procedure, we had to reformulate in $\S 2$ the most part of the results in the above quoted papers to the present situation in which the vector field $F$ is not compact but only condensing. In this way we obtained results which are interesting in themselves besides being necessary for the proof of the main existence Theorem 2.1 and its straightforward consequence Theorem 2.2 .

In particular, we would like to mention Lemma 2.4, which states the existence of zeros for Lefschetz w-carrier with nonzero index, verifying the Borsuk Ulam condition on the relative boundary (with respect to an open ball) of a compact, convex set $K \subset \mathbb{R}^{n}$ which is symmetric with respect to the origin. We would like also to point out that here we use the theory of w-carrier instead of that of $\mathrm{W}$-maps as in [4, 5 and 10$]$.

In $\S 3$ we will present two applications of our abstract results. Specifically, first we will consider a controlled neutral functional differential equation on a given finite time interval and, by using Theorem 2.1 , we will solve the problem of finding a control law in a suitable finite-dimensional control space in such a way that the corresponding trajectory reaches at the final time a given target set.

In the second example, in order to apply Theorem 2.3 , we will deal with a semilinear differential inclusion in a Banach space and we will give sufficient conditions to show that it has periodic solutions of a prescribed period.

In the next section, $\S 1$, we give the definitions and some known results we will need through the paper.

\section{DEFINITIONS AND PRELIMINARY RESULTS}

Let $X, Y$ be topological spaces; a multivalued map $M$ from $X$ into $Y$ will be denoted by the symbol $M: X \multimap \circ Y$.

Definition 1.1. A multivalued map $M: X-\circ Y$ is said to be upper semicontinuous (shortly u.s.c.) if the set 


$$
M^{-1}(V)=\{x \in X: M(x) \subset V\}
$$

is open in $X$ for every open $V \subset Y$.

It is not difficult to verify the following properties (see, e.g., [2]).

Lemma 1.1. (a) If $M_{1}: X-\circ Y, M_{2}: Y \rightarrow \circ Z$ are u.s.c. multivalued maps then their composition $M_{2} \circ M_{1}: X-\circ Z$,

$$
\left(M_{2} \circ M_{1}\right)(x)=M_{2}\left(M_{1}(x)\right)
$$

is an u.s.c. multivalued map.

(b) If $M_{1}: X \multimap Y_{1}, M_{2}: X \multimap Y_{2}$ are u.s.c. multivalued maps with compact values then their Cartesian product $M_{1} \times M_{2}: X-\circ Y_{1} \times Y_{2}$,

$$
\left(M_{1} \times M_{2}\right)(x)=M_{1}(x) \times M_{2}(x)
$$

is an u.s.c. multivalued map.

Definition 1.2. A multivalued map $M: X \multimap Y$ is said to be closed if its graph

$$
\operatorname{Gr} M=\{(x, y) \in X \times Y: y \in M(x)\}
$$

is a closed subset of $X \times Y$.

If a multivalued map $M: X-\circ Y$ is closed and compact (i.e. $M(X)$ is relatively compact in $Y$ ) then $M$ is upper semicontinuous.

By an $\varepsilon$-neighborhood of a subset $\Omega$ of a metric space $X$ we mean the set

$$
\varepsilon \Omega=\{y \in X: \exists x \in \Omega \text { such that } d(x, y)<\varepsilon\} .
$$

Definition 1.3. Let $X, Y$ be metric spaces and let $M: X \multimap Y$ be a multivalued map. A multivalued map $M^{\prime}: X-\circ Y$ is said to be an $\varepsilon$-approximation of $M$ if $\operatorname{Gr} M^{\prime} \subset \varepsilon \operatorname{Gr} M$.

The following result is due to A. Lasota and Z. Opial [9].

Theorem 1.1. Let $X$ be a metric space and $Y$ a metric locally convex space. Then for every $\varepsilon>0$ any u.s.c. multivalued map $M: X-\circ Y$ with closed, convex values has a single-valued continuous $\varepsilon$-approximation

$$
\mu: X \rightarrow Y \text { such that } \mu(X) \subset \operatorname{co} M(X) .
$$

Definition 1.4 (cf. [6]). Let $X$ and $Y$ be Hausdorff topological spaces. A multivalued u.s.c. map $M: X-\circ Y$ with compact values is said to be a weighted carrier / (w-carrier) if, for any $x \in X$, to any piece $C$ (i.e. open and closed subset) of $M(x)$ is assigned a weight or multiplicity $m(C, M(x)) \in \mathbb{Z}$ in such a way that the following properties hold:

(a) $m(\cdot, M(x))$ is an additive function, i.e.

$$
m\left(C_{1} \cup C_{2}, M(x)\right)=m\left(C_{1}, M(x)\right)+m\left(C_{2}, M(x)\right)
$$

whenever $C_{1} \cap C_{2}=\varnothing$;

(b) if $U$ is open in $Y$ with $\partial U \cap M(x)=\varnothing$, then $m(M(x) \cap U, M(x))=$ $m\left(M\left(x^{\prime}\right) \cap U, M\left(x^{\prime}\right)\right)$ whenever $x^{\prime}$ is close enough to $x$.

Definition 1.5 (cf. [6]). If $U \subset Y$ is an open set, $M(x) \cap \partial U=\varnothing$, then the number $i(M(x), U)=m(M(x) \cap U, M(x))$ is called the index or multiplicity of $M(x)$ in $U$. 
If $X$ is connected the number $i(M(x), U)$ does not depend on $x \in X$. In this case the number $i(M)=i(M(x), Y)$ will be called the index of the w-carrier $M$.

Let us mention the following properties of w-carriers (see [6]).

(1) Let $M: X \multimap \circ Y$ be an u.s.c. multivalued map with compact, connected values. Then $M$ becomes a w-carrier by assigning multiplicity 1 to $M(x)$. In particular every continuous single-valued $f$ is a w-carrier with $i(f)=1$.

(2) Let $M: X \multimap Y$ be a w-carrier and $f: Z \rightarrow X$ (resp. $f^{\prime}: Y \rightarrow W$ ) be a single-valued continuous map. Then $M \circ f: Z \multimap Y$ (resp. $f^{\prime} \circ M: X \multimap W$ ) is a w-carrier.

(3) Let $M: X-\circ Y$ be a w-carrier and $f: Z \rightarrow W$ be a continuous single-valued map. Then $M \times f: X \times Z-\circ Y \times W$ is a w-carrier.

(4) Let $M: X-\circ Y$ be a w-carrier, then the graph map $\Gamma_{F}: X-\circ X \times Y$, $\Gamma_{F}(x)=\{x\} \times M(x)$ is a w-carrier.

By a weighted map (w-map) we mean any w-carrier $M: X-\circ Y$ such that $M(x)$ is a finite subset of $Y$ for every $x \in X$. D.

Let $\mathrm{H}(D)=\left\{\mathrm{H}_{\mathbf{q}}(D)\right\}_{q \geq 0}$ be the graded Čech homology module of a space

Definition 1.6 (cf. [6]). A w-carrier $M: X \multimap Y$ is said to be acyclic if for every $x \in X$,

$$
\mathrm{H}_{\mathrm{q}}(M(x))=0 \text { for all } q>0
$$

Following [6] we may define the notion of Lefschetz w-carrier.

Definition 1.7. A w-carrier $M: X-\circ Y$ is said to be a Lefschetz w-carrier if it can be factorized in the form<smiles>[Y]O[Te]</smiles>

where $Q: X-\circ Z$ is an acyclic w-carrier and $r: Z \rightarrow Y$ is a continuous single-valued map.

Definition 1.8. Let $X$ be a topological vector space; let $\mathscr{A} \subset X$ be a set symmetric with respect to the origin. We will say that a multivalued map $L: \mathscr{A} \longrightarrow \mathrm{X}$ verifies the Borsuk-Ulam property on $\mathscr{A}$ if

(B.U.) for each $x \in \mathscr{A}$ the sets $L(x)$ and $L(-x)$ are strictly separated by a hyperplane, that is, for each $x \in \mathscr{A}$ there exists a continuous functional $x^{*} \in X^{*}$, the dual of $X$, such that $x^{*}(y)>0$ for every $y \in L(x)$ and $x^{*}(y)<0$ for every $y \in L(-x)$.

Using the methods illustrated in the papers $[6,10]$ one can prove the following generalization of Theorem 2.8 of [10].

Theorem 1.2. Let $B$ be the closed unit ball in $\mathbb{R}^{n}$. Let $L: B-\circ \mathbb{R}^{n}$ be a Lefschetz $w$-carrier with $i(L) \neq 0$. If $L$ verifies the (B.U.) property on $\partial B$ then there exists $x \in \stackrel{\circ}{B}$ such that $0 \in L(x)$.

Let us recall the following notions (see, for example, $[1,16]$ ) 
Definition 1.9. Let $X$ be a Banach space; a function $\psi: 2^{X} \rightarrow[0,+\infty)$ is said to be a measure of noncompactness in $X$ if $\psi(\overline{c o} \Omega)=\psi(\Omega)$ for every $\Omega \in 2^{X}$. A measure of noncompactness $\psi$ is called

(i) monotone if $\Omega_{0}, \Omega_{1} \in 2^{X}$ and $\Omega_{0} \subset \Omega_{1}$ imply $\psi\left(\Omega_{0}\right) \leq \psi\left(\Omega_{1}\right)$;

(ii) nonsingular if $\psi(\{a\} \cup \Omega)=\psi(\Omega)$ for every $a \in X$ and $\Omega \in 2^{X}$; $2^{X}$

(iii) semiadditive if $\psi\left(\Omega_{0} \cup \Omega_{1}\right)=\max \left\{\psi\left(\Omega_{0}\right), \psi\left(\Omega_{1}\right)\right\}$ for every $\Omega_{0}, \Omega_{1} \in$

(iv) algebraically semiadditive if $\psi\left(\Omega_{0}+\Omega_{1}\right) \leq \psi\left(\Omega_{0}\right)+\psi\left(\Omega_{1}\right)$;

(v) regular if $\psi(\Omega)=0$ is equivalent to the relative compactness of $\Omega$;

(vi) invariant with respect to the reflection at the origin if $\psi(-\Omega)=\psi(\Omega)$ for every $\Omega \in 2^{X}$.

Well-known examples of measures of noncompactness satisfying the properties (i)-(vi) are: the Kuratowski measure of noncompactness

$\alpha(\Omega)=\inf \{d>0: \Omega$ admits a partition into a finite number of sets whose diameters are less than $d\}$

and the Hausdorff measure of noncompactness

$$
\chi(\Omega)=\inf \{\varepsilon>0: \Omega \text { has a finite } \varepsilon \text {-net }\} .
$$

Definition 1.10. Let $X_{0}$ be a closed subset of a Banach space $X ; \psi$ be a measure of noncompactness in $X$ and $0 \leq k<1$. A multivalued map $\mathscr{F}$ : $X_{0} \multimap \circ X$ with compact values is said to be $(k, \psi)$-condensing if $\psi(\mathscr{F}(\Omega)) \leq$ $k \psi(\Omega)$ for every $\Omega \subset X$. Let $\Lambda$ be a topological space, then a multivalued map $G: \Lambda \times X_{0}-\circ X$ is said to be $(k, \psi)$-condensing in the second variable if $\psi(G(K \times \Omega)) \leq k \psi(\Omega)$ for every compact $K \subset \Lambda$ and $\Omega \subset X$.

Definition 1.11 (cf. [1, 11]). A closed, convex set $\mathscr{M} \subset X$ is called fundamental for a multivalued map $\mathscr{F}: X_{0} \multimap \circ X$ if

(1) $\mathscr{F}\left(X_{0} \cap \mathscr{M}\right) \subseteq \mathscr{M}$,

(2) $x_{0} \in \overline{\operatorname{co}}\left(\mathscr{F}\left(x_{0}\right) \cup \mathscr{M}\right)$ implies $x_{0} \in \mathscr{M}$.

A closed convex set $\mathscr{M} \subset X$ is called fundamental for a family $G: \Lambda \times X_{0} \multimap$ $X$ if it is fundamental for every multivalued map $G(\lambda, \cdot), \lambda \in \Lambda$. Finally, a fundamental set $\mathscr{M}$ of a multivalued map $\mathscr{F}$ is called essential if it is compact and $X_{0} \cap \mathscr{M} \neq \varnothing$.

Following the methods presented in [1 and 14] one can prove the following statements.

Lemma 1.2. Let $X_{0}$ be a closed subset of a Banach space $X, \Lambda$ be a topological space, let $G: \Lambda \times X_{0} \rightarrow X$ be a closed multivalued map which is $(k, \psi)$ condensing in the second variable where $0 \leq k<1$ and $\psi$ is a regular monotone nonsingular measure of noncompactness in $X$. Then for every compact $K \subset \Lambda$ there exists an essential fundamental set of the family $\left.G\right|_{K \times X_{0}}$.

Lemma 1.3. Let $X_{0}$ be a closed subset of a Banach space $X, \mathscr{F}: X_{0} \rightarrow \circ X$ be a closed multivalued map which is $(k, \psi)$-condensing where $0 \leq k<1$ and the measure of noncompactness $\psi$ is regular, monotone, nonsingular, semiadditive and invariant with respect to the reflection at the origin. Then $\mathscr{F}$ has an essential fundamental set which is symmetric with respect to the origin.

Let $V$ be an open bounded subset of a Banach space $Y$, let $\psi$ be a regular monotone nonsingular measure of noncompactness in $Y, 0 \leq k<1$, and 
$\bar{F}: \bar{V}$ - $Y$ be a closed $(k, \psi)$-condensing multivalued map with convex values such that $y \notin \overline{\mathscr{F}}(y)$ for all $x \in \partial V$. Then the topological degree of the corresponding multivalued vector field $\mathscr{F}, \mathscr{F}(x)=x-\overline{\mathscr{F}}(x)$, can be defined by the formula

$$
\operatorname{Deg}(\mathscr{F}, V, 0)=\operatorname{Deg}_{\mathscr{M}}\left(\mathscr{F}, V_{\mathscr{M}}, 0\right)
$$

where $\mathscr{M}$ is an arbitrary essential fundamental set of $\overline{\mathscr{F}}$ and $\operatorname{Deg}_{\mathscr{M}}$ denotes the relative topological degree of a compact multivalued vector field (see $[1,11$, 14]). Let us remember that in turn the relative degree $\operatorname{Deg}_{\mathscr{M}}\left(\mathscr{F}, V_{\mathscr{M}}, 0\right)$ can be defined as $\operatorname{Deg}_{\mathscr{M}}\left(i-\bar{f}, V_{\mathscr{M}}, 0\right)$, where $\bar{f}$ is an arbitrary $\varepsilon$-approximation of $\left.\bar{F}\right|_{\bar{V}}$ and $\varepsilon$ is sufficiently small (see [1]).

We will need the following properties of the introduced topological degree.

(1) The fixed point property. If $\operatorname{Deg}(\mathscr{F}, V, 0) \neq 0$ then the fixed point set Fix $\overline{\mathscr{F}}=\{y: y \in \overline{\mathscr{F}}(y)\}$ is nonempty and compact.

(2) The generalized topological invariance. Let $\Lambda$ be a path connected topological space, $\bar{G}: \Lambda \times \bar{V}$ — $Y$ be a closed $(k, \psi)$-condensing in the second variable multivalued map $(0 \leq k<1)$. If $y \notin \bar{G}(\lambda, y)$ for all $\lambda \in \Lambda$ and $y \in \partial V$ then

$$
\operatorname{Deg}\left(G\left(\lambda_{0}, \cdot\right), V, 0\right)=\operatorname{Deg}\left(G\left(\lambda_{1}, \cdot\right), V, 0\right)
$$

for all $\lambda_{0}, \lambda_{1} \in \Lambda$, where $G(\lambda, y)=y-\bar{G}(\lambda, y)$.

(3) The additivity property. If $V=V_{1} \cup V_{2}$ where $V_{1}$ and $V_{2}$ are open and disjoint and $y \notin \overline{\mathscr{F}}(y)$ for all $y \in \partial V_{1} \cup \partial V_{2}$ then

$$
\operatorname{Deg}(\mathscr{F}, V, 0)=\operatorname{Deg}\left(\mathscr{F}, V_{1}, 0\right)+\operatorname{Deg}\left(\mathscr{F}, V_{2}, 0\right) .
$$

(4) The index of a fixed point. If $y \in V$ is an isolated fixed point of $\bar{F}$ then its index $\operatorname{ind}(\bar{F}, y)$ is defined as $\operatorname{Deg}(\mathscr{F}, \Omega, 0)$ where $\Omega$ is a neighborhood of $y$ which does not contain other fixed points of $\overline{\mathscr{F}}$. If $\overline{\mathscr{F}}$ has in $V$ only a finite number of isolated fixed points $y_{1}, \ldots, y_{n}$ then

$$
\operatorname{Deg}(\mathscr{F}, V, 0)=\sum_{i=1}^{n} \operatorname{ind}\left(\overline{\mathscr{F}}, y_{i}\right) .
$$

Using results of the paper [7] one may prove the following statement.

Lemma 1.4. Let $\Lambda$ be a topological space, $Y_{0}$ be a closed subset of a Banach space $Y, \psi$ be a regular monotone measure of noncompactness in $Y$. Let $\bar{G}: \Lambda \times Y_{0} \longrightarrow Y$ be a closed $(k, \psi)$-condensing in the second variable multivalued map $(0 \leq k<1)$. If for every $\lambda \in \Lambda$ the set $S(\lambda)=\left\{y \in Y_{0}: y \in \bar{G}(\lambda, y)\right\}$ is nonempty then the multivalued map $S: \lambda-\circ Y_{0}$ is compact-valued and u.s.c.

Definition 1.12 (cf. [8]). Let $X, Y$ be metric spaces. An u.s.c. multivalued map $M: X \multimap \circ Y$ is said to be admissible if there are maps $Q_{i}: Z_{i} \multimap \circ Z_{i+1}$, $i=0,1, \ldots, m\left(Z_{i}\right.$ metric spaces, $\left.Z_{0}=X, Z_{m+1}=Y\right)$, satisfying

(a) $M=Q_{m} \circ \cdots \circ Q_{0}$

(b) $Q_{i}$ is u.s.c. with acyclic, compact values for each $i=0,1, \ldots, m$.

Let us note an important particular case

Definition 1.13. If in the previous definition $m=1$ and $Q_{1}=q$ is a continuous single-valued map then the map $M=q \circ Q_{0}$ will be called permissible. 
Definition 1.14. Let $U \subset X, V \subset Y$ be open sets, where $X, Y$ are metric spaces. Let $\bar{F}: \bar{U} \times \bar{V}-\circ Y$ be a multivalued map and let $F(x, y)=y-$ $\bar{F}(x, y)$. We shall denote by

$$
S^{F}=\{(x, y) \in \bar{U} \times \bar{V}: y \in \bar{F}(x, y)\}, \quad \mathscr{D}^{F}=\left\{x \in X: S_{x}^{F} \subset U \times V\right\},
$$

where $S_{A}^{F}=S^{F} \cap(A \times Y)$ for any $A \subset X$.

\section{RESULTS}

We want to prove the existence of solutions for the system

$$
\left\{\begin{array} { l } 
{ y \in \overline { F } ( x , y ) } \\
{ x \in \overline { G } ( x , y ) }
\end{array} \text { or equivalently } \left\{\begin{array}{l}
0 \in y-\bar{F}(x, y)=F(x, y) \\
0 \in x-\bar{G}(x, y)=G(x, y) .
\end{array}\right.\right.
$$

For this, let $X, Y$ be Banach spaces, $\psi_{X}$ and $\psi_{Y}$ be regular monotone nonsingular measures of noncompactness in $X$ and $Y$ respectively; the measure $\psi_{X}$ is also semiadditive and invariant with respect to the reflection at the origin. Let $U \subset X$ be an open neighborhood of the origin and $V \subset Y$ be an open set.

We can now formulate the following.

Theorem 2.1. Let $\bar{F}: \bar{U} \times \bar{V} \multimap Y$ be a closed $\left(k, \psi_{Y}\right)$-condensing in the second variable, where $0 \leq k<1$, multivalued map with convex values and $\bar{G}: \bar{U} \times$ $\bar{V} \multimap X$ be a permissible multivalued map. Suppose that there exists $r>0$ such that $\bar{B}(0, r) \subset \bar{U} \cap \mathscr{D}^{F}$ and $\operatorname{Deg}(F(0, \cdot), V, 0) \neq 0$. Let $T: \bar{B}(0, r) \multimap X$ be the operator defined by $T(x)=x-\bar{T}(x)$, where $\bar{T}(x)=\bar{G}(x, S(x))$ and $S(x)=$ $\{y \in Y: y \in \bar{F}(x, y)\}$. Suppose that the multivalued map $\widehat{G}: \bar{B}(0, r)-\circ X$, given by $\widehat{G}(x)=\bar{G}(x, \bar{V})$ is closed and $\left(l, \psi_{X}\right)$-condensing, where $0 \leq l<1$, and that for every $x \in \partial B(0, r)$ such that $0 \notin T(x)$, the sets $T(x)$ and $T(-x)$ are strictly separated by a hyperplane.

The system (2.1) has a solution.

The proof of Theorem 2.1 needs several preliminary results. First of all, observe that the multivalued map $\bar{T}$ has nonempty, compact values and it is upper semicontinuous. These facts follow from the assumption that

$$
\operatorname{Deg}(F(0, \cdot), V, 0) \neq 0
$$

and Lemmas 1.1 and 1.4.

Moreover, Lemma 1.3 states that the multivalued map $\widehat{G}$ has a symmetric (with respect to the origin) essential fundamental set $\mathscr{H} \subset H$. Let $\bar{B}_{\mathscr{L}}=$ $\bar{B}(0, r) \cap \mathscr{H}$ and let $\mathscr{M} \subset Y$ be an essential fundamental set of the family $\left.\bar{F}\right|_{\bar{B}_{\mathscr{X}} \times \bar{V}}$.

Let $V_{\mathscr{M}}=V \cap \mathscr{M}$ and let $\bar{V}_{\mathscr{M}}, \partial V_{\mathscr{M}}$ denote the closure and the boundary of $V_{\mathscr{K}}$ respectively in the relative topology of the space $\mathscr{M}$. It is easy to see that $S_{\bar{B}_{\mathscr{R}}}^{F} \subset \bar{B}_{\mathscr{H}} \times V_{\mathscr{M}}$.

First of all we need the following extension of Lemma 2.2 of [4].

Lemma 2.1. For each neighborhood $W \subset \bar{B}_{\mathscr{L}} \times V_{\mathscr{K}}$ (in the relative topology) of the set $S_{\bar{B}_{\mathscr{H}}}$, there exists $\varepsilon>0$ such that if $\bar{f}: \bar{B}_{\mathscr{H}} \times \bar{V}_{\mathscr{M}} \rightarrow Y$ is an $\varepsilon$-approximation of $\left.\bar{F}\right|_{\bar{B}_{\mathscr{X}} \times \bar{V}_{\mathscr{X}}}$, then $S_{\bar{B}_{\mathscr{X}}}^{f} \subset W$. 
Proof. Since the map $\bar{F}$ is closed the set $S_{\bar{B}_{\mathscr{X}}}^{F}$ is compact. Let $W_{1} \subset \bar{B}_{\mathscr{H}} \times \bar{V}_{\mathscr{K}}$ be an $\varepsilon_{1}$-neighborhood of $S_{B_{X}}^{F}$ such that $W_{1} \subset W$ and $\partial W_{1} \cap \partial W=\varnothing$. Let $\varepsilon_{2}=\operatorname{dist}\left(\partial W, \partial W_{1}\right)=\min \left\{d\left(w, w_{1}\right): w \in \partial W, w_{1} \in \partial W_{1}\right\}$ and $A=$ $\left(\bar{B}_{\mathscr{H}} \times \bar{V}_{\mathscr{K}}\right) \backslash W_{1}$. Then $F(A) \subset Y$ is a closed set, $0 \notin F(A)$ and let $\varepsilon_{3}>0$ be such that $B\left(0, \varepsilon_{3}\right) \subset Y, \bar{B}\left(0, \varepsilon_{3}\right) \cap F(A)=\varnothing$. Hence, if $(x, y) \in A$ then $\operatorname{dist}(y, \bar{F}(x, y))>\varepsilon_{3}$. Let $\varepsilon=\min \left\{\varepsilon_{1}, \varepsilon_{2}, \varepsilon_{3}\right\}$ and let $\bar{f}: \bar{B}_{\mathscr{H}} \times \bar{V}_{\mathscr{K}} \rightarrow Y$ by an $\varepsilon$-approximation of $\left.\bar{F}\right|_{\bar{B}_{\mathscr{X}} \times \bar{V}_{\mathscr{M}}}$. Let $(x, y) \in\left(\bar{B}_{\mathscr{H}} \times \bar{V}_{\mathscr{M}}\right) \backslash W$ and let $y=\bar{f}(x, y)$, that is $(x, y) \in S_{\bar{B}_{\mathscr{X}}}^{f}$. Then there exists $(\bar{x}, \bar{y}) \in \bar{B}_{\mathscr{L}} \times \bar{V}_{\mathscr{M}}$ such that $\|(x, y)-(\bar{x}, \bar{y})\|+\|\bar{f}(x, y)-z\|<\varepsilon$ for some $z \in \bar{F}(\bar{x}, \bar{y})$. As $\|(x, y)-(\bar{x}, \bar{y})\|<\varepsilon$ it follows that $(\bar{x}, \bar{y}) \notin S_{\bar{B}_{\mathscr{X}}}$. Since $y=\bar{f}(x, y)$ we get

$$
\|\bar{y}-z\| \leq\|y-\bar{y}\|+\|y-z\| \leq\|(x, y)-(\bar{x}, \bar{y})\|+\|\bar{f}(x, y)-z\|<\varepsilon .
$$

Hence $(\bar{x}, \bar{y}) \notin A$. Thus $(\bar{x}, \bar{y}) \in W_{1} \backslash S_{\bar{B}_{\mathscr{X}}}^{F}$. This is an absurd, since $(x, y) \in$ $\left(\bar{B}_{\mathscr{H}} \times \bar{V}_{\mathscr{M}}\right) \backslash W$ and $\|(x, y)-(\bar{x}, \bar{y})\|<\varepsilon$ and so $(\bar{x}, \bar{y}) \notin W_{1}$.

Following the same arguments as in Lemma 2.3 of [4], we may obtain the following result.

Lemma 2.2. Let $X=\mathbb{R}^{n}, Y=\mathbb{R}^{m}$. There exists an $\varepsilon_{0}>0$ such that for all $\varepsilon<\varepsilon_{0}$ there exists a single-valued continuous map $\bar{f}: \bar{U} \times \bar{V} \rightarrow \mathbb{R}^{m}$ such that $\bar{f}: \bar{B}_{\mathscr{H}} \times \bar{V}_{\mathscr{M}} \rightarrow \mathscr{M}$ is an E-approximation of $\left.\bar{F}\right|_{\bar{B}_{\mathscr{X}} \times \bar{V}_{\mathscr{Z}}}$ and

(a) for every $x \in \bar{B}_{\mathscr{X}}$ the set $S^{f}(x)=\{y \in Y: y=\bar{f}(x, y)\}$ is a finite subset of $V$;

(b) $\operatorname{Deg}(F(0, \cdot), V, 0)=\operatorname{Deg}_{\mathscr{M}}\left(f(0, \cdot), V_{\mathscr{M}}, 0\right)$.

Lemma 2.3. Let $X, Y$ and $\bar{F}$ satisfy the conditions of Theorem 2.1. Let us suppose that for all $x \in \bar{B}_{\mathscr{Z}}$ the inclusion $y \in \bar{F}(x, y)$ has only isolated solutions. Then the multivalued map $S: \bar{B}_{\mathscr{C}}-\circ V_{\mathscr{M}}$ is a w-map and $i(S)=\operatorname{Deg}(F(0, \cdot), V, 0)$.

Proof. The upper semicontinuity of the multivalued map $S$ follows from Lemma 1.4. If $y \in S(x)$ then $y$ is an isolated fixed point of $\bar{F}(x, \cdot)$ and we can put $m(y, S(x))=\operatorname{ind}(\bar{F}(x, \cdot), y)$. Now, let $\Omega$ be an open set in $V$ such that $S(x) \cap \partial \Omega_{\mathscr{M}}=\varnothing$, where $\Omega_{\mathscr{K}}=\Omega \cap \mathscr{M}$. From the upper semicontinuity of $S$ it follows that there exists a ball $\bar{B}(x, \delta)$ such that $S(\bar{B}(x, \delta) \cap \mathscr{H}) \cap \partial \Omega_{\mathscr{M}}=\varnothing$. Then, for every $x^{\prime} \in \bar{B}(x, \delta) \cap \mathscr{H}$, the admissible homotopy $H:[0,1] \times$ $\bar{\Omega}_{\mathscr{M}} \longrightarrow \mathscr{M}$, given by

$$
H(\lambda, y)=\bar{F}\left(\lambda x+(1-\lambda) x^{\prime}, y\right)
$$

ensures that

$$
\operatorname{Deg}_{\mathscr{M}}(F(x, \cdot), \Omega, 0)=\operatorname{Deg}_{\mathscr{M}}\left(F\left(x^{\prime}, \cdot\right), \Omega, 0\right) .
$$

Therefore

$$
\begin{aligned}
m(S(x) \cap \Omega, S(x)) & =\sum_{y \in S(x) \cap \Omega} \operatorname{ind}(\bar{F}(x, \cdot), y)=\operatorname{Deg}_{\mathscr{M}}(F(x, \cdot), \Omega, 0) \\
& =\operatorname{Deg}_{\mathscr{M}}\left(F\left(x^{\prime}, \cdot\right), \Omega, 0\right) \\
& =\sum_{y^{\prime} \in S\left(x^{\prime}\right) \cap \Omega} \operatorname{ind}\left(\bar{F}\left(x^{\prime}, \cdot\right), y^{\prime}\right)=m\left(S\left(x^{\prime}\right) \cap \Omega, S\left(x^{\prime}\right)\right) .
\end{aligned}
$$


Thus $S$ is a w-map and

$$
i(S)=i\left(S(0), V_{\mathscr{M}}\right)=\sum_{y \in S(0)} \operatorname{ind}(\bar{F}(0, \cdot), y)=\operatorname{Deg}(F(0, \cdot), V, 0) .
$$

In the sequel we need the following result which is the "relative" version of Theorem 1.2.

Lemma 2.4. Let $K \subset \mathbb{R}^{n}$ be a convex, compact set, symmetric with respect to the origin, let $\bar{B}_{K}=\bar{B}(0, r) \cap K$. Let $L: \bar{B}_{K} \rightarrow \circ \mathbb{R}^{n}$ be a Lefschet $z$-carrier such that $i(L) \neq 0$ and $\bar{L}\left(\bar{B}_{K}\right) \subset K$, where $\bar{L}(x)=x-L(x)$. If $L$ verifies the (B.U.) condition on $\partial \bar{B}_{K}=\partial B(0, r) \cap K$, then there exists $x \in B_{K}$ such that $0 \in L(x)$.

Proof. Let $\rho: \mathbb{R}^{n} \rightarrow K$ be any retraction, then the mapping $\tilde{\rho}: \mathbb{R}^{n} \rightarrow K$, defined by $\tilde{\rho}(x)=\frac{1}{2}[\rho(x)-\rho(-x)]$ is an odd retraction. Then the set $\tilde{\rho}^{-1}\left(\bar{B}_{K}\right)$ is symmetric with respect to the origin. Passing, if necessary, to its connected component containing $\bar{B}_{K}$ and using the Dugundji's extension theorem we may assume without loss of generality that $\tilde{\rho}^{-1}\left(\bar{B}_{K}\right)$ and $\mathbb{R}^{n} \backslash \tilde{\rho}^{-1}\left(\bar{B}_{K}\right)$ are connected sets. Now, let $B_{1}=B\left(0, r_{1}\right) \subset \mathbb{R}^{n}$ be an open ball containing the set $K$. Let us consider the multivalued map $\tilde{L}: D \multimap \circ \mathbb{R}^{n}$, given by $\tilde{L}(x)=x-\bar{L} \circ \tilde{\rho}(x)$ with $D=\bar{B}_{1} \cap \tilde{\rho}^{-1}\left(\bar{B}_{K}\right)$. The map $\tilde{L}$ is a Lefschetz w-carrier since it can be decomposed in the form

$$
D \stackrel{\Gamma}{\longrightarrow} \mathbb{R}^{n} \times \mathbb{R}^{n} \stackrel{d}{\longrightarrow} \mathbb{R}^{n}
$$

where $\Gamma=\Gamma_{\bar{L} \circ \tilde{\rho}}$ is the graph map and $d(x, y)=x-y$. Moreover, $i(\widetilde{L})=$ $i(L) \neq 0$. Let us show now that $\widetilde{L}$ verifies the (B.U.) condition on $\partial D$. In fact, if $x \in \partial D$ and $x \in \partial B_{K}$ the assertion follows from the fact that $\left.\widetilde{L}\right|_{\partial B_{K}}=\left.L\right|_{\partial B_{K}}$ and our assumptions.

Let $x \in \partial B_{1} \cap \tilde{\rho}^{-1}\left(\bar{B}_{K}\right)$ then by Proposition 2.10 of [10] it is sufficient to show that

$$
0 \notin k(\overline{\mathrm{co}} \tilde{L}(x))-\overline{\mathrm{co}} \tilde{L}(-x),
$$

where $k(A)=\{\lambda x: \lambda \in[0,1], x \in A\}$. Assume, to the contrary, that there exist $\lambda_{0} \in[0,1], y_{1} \in \overline{\operatorname{co}}(\bar{L} \circ \tilde{\rho}(x)) \subset K$ and $y_{2} \in \overline{\operatorname{co}}(\bar{L} \circ \tilde{\rho}(-x)) \subset K$ such that $\lambda_{0}\left(x-y_{1}\right)=-x-y_{2}$. Then $\left(\lambda_{0}+1\right)\|x\|=\left\|\lambda y_{1}-y_{2}\right\|<(\lambda+1) r_{1}$. This contradicts the fact that $x \in \partial B_{1}$. Finally from Theorem 1.2 follows the existence of a point $x \in D$ such that $0 \in \widetilde{L}(x)$, that is $x \in \bar{L} \circ \tilde{\rho}(x) \subset K$, hence $\tilde{\rho}(x)=x$ and $0 \in L(x)$.

Let us observe that we do not exclude the case when $K \subset B(0, r), \partial B_{K}=\varnothing$. In this case we have the following

Corollary 2.1 (cf. [6]). Let $K \subset \mathbb{R}^{n}$ be a convex compact set, $\bar{L}: K-\circ K$ be a Lefschetz $w$-carrier, $i(\bar{L}) \neq 0$. Then $\bar{L}$ has a fixed point.

We are now in the position of proving Theorem 2.1 .

Proof. Let $T_{\mathscr{H}}=\left.T\right|_{\bar{B}_{\mathscr{X}}}$. Assume to the contrary that system (1) does not have solutions in the set $\bar{B}_{\mathscr{H}}$. Then $0 \notin T_{\mathscr{H}}(x)$ for all $x \in \bar{B}_{\mathscr{H}}$. Since $T_{\mathscr{H}}$ is an 
u.s.c. multivalued map, this assumption implies the existence of an $\varepsilon_{1}>0$ such that $B\left(0, \varepsilon_{1}\right) \cap T_{\mathscr{H}}\left(\bar{B}_{\mathscr{H}}\right)=\varnothing$.

On the other hand from the Proposition 2.13 of [10] it follows that there exists $\varepsilon_{2}>0$ such that every $\varepsilon_{2}$-approximation $T^{\prime}: \bar{B}_{\mathscr{H}}-\circ X$ of $T_{\mathscr{H}}$ verifies the (B.U.) property on $\partial B_{\mathscr{H}}$.

Let $\delta=\min \left\{\varepsilon_{1}, \varepsilon_{2}\right\}$ and let $W \subset \bar{B}_{\mathscr{H}} \times V_{\mathscr{M}}$, given by

$$
W=\left\{(x, y) \in \bar{B}_{\mathscr{H}} \times V_{\mathscr{M}}:(x, G(x, y)) \in \delta \mathrm{Gr} T_{\mathscr{H}}\right\} .
$$

It is clear that $W$ is an open neighborhood (in the relative topology) of the set $S_{\bar{B}_{\boldsymbol{X}}}$

We will divide the rest of the proof into three parts.

First step. $X=\mathbb{R}^{n}, Y=\mathbb{R}^{m}$.

Let $\varepsilon^{*}>0$ be that one given by Lemma 2.1 , i.e. every $\varepsilon^{*}$-approximation $\bar{f}$ of $\left.\bar{F}\right|_{\bar{B}_{\mathscr{X}} \times \bar{V}_{\mathscr{R}}}$ has the property that $S_{\bar{B}_{\mathscr{X}}}^{f} \subset W$.

Let $\varepsilon_{0}$ be given by Lemma 2.2 and let $\varepsilon^{\prime}=\min \left\{\varepsilon^{*}, \varepsilon_{0}, \delta\right\}$. By Lemmas 2.2 and 2.3 there exists an $\varepsilon^{\prime}$-approximation $\bar{f}^{\prime}$ of $\left.\bar{F}\right|_{\bar{B}_{\mathscr{X}} \times \bar{V}_{\mathscr{K}}}$ such that the map $S^{\prime}: \bar{B}_{\mathscr{L}} \longrightarrow V_{\mathscr{M}}$ defined by $S^{\prime}(x)=\left\{y: y=\bar{f}^{\prime}(x, y)\right\}$ is a w-map. The index of this map is given by

$$
\begin{aligned}
i\left(S^{\prime}\right) & =\operatorname{Deg}\left(f^{\prime}(0, \cdot), W(0), 0\right) \\
& =\operatorname{Deg}(F(0, \cdot), W(0), 0)=\operatorname{Deg}(F(0, \cdot), V, 0) \neq 0 .
\end{aligned}
$$

Now let us show that the multivalued map $\bar{T}^{\prime}(x)=\bar{G}\left(x, S^{\prime}(x)\right)$ is a Lefschetz w-carrier. Indeed, since the map $\bar{G}$ is permissible it can be represented as $\bar{G}=q \circ Q$ where $Q: \bar{B}_{\mathscr{H}}-\circ Z$ is acyclic-valued and $q: Z \rightarrow \mathbb{R}^{n}$ is singlevalued. Then $\bar{T}^{\prime}$ may be decomposed as

$$
\bar{B}_{\mathscr{H}} \stackrel{\mathscr{G}}{\longrightarrow} Z \times \mathbb{R}^{m} \stackrel{r_{1}}{\longrightarrow} Z \stackrel{q}{\longrightarrow} \mathbb{R}^{n}
$$

where $\mathscr{G}(x)=\bigcup_{y \in S^{\prime}(x)}(Q(x, y) \times\{y\})$ is an acyclic w-carrier and $r_{1}$ is a natural projection. Moreover, $i\left(T^{\prime}\right)=i\left(\bar{T}^{\prime}\right)=i\left(S^{\prime}\right) \neq 0$.

Since $S_{\bar{B}_{\mathscr{X}}}^{f^{\prime}} \subset W$ we have that $\operatorname{Gr} T^{\prime} \subset \delta \mathrm{Gr} T_{\mathscr{H}}$, i.e. $T^{\prime}$ is a $\delta$-approximation of $T_{\mathscr{H}}$. From our choice of $\delta$ it follows that $T^{\prime}$ satisfies the B.U. condition on $\partial B_{\mathscr{K}}$. Hence, from Lemma 2.4 it follows that there exists $x \in B_{\mathscr{K}}$ such that $0 \in T^{\prime}(x)$. Then $0 \in \delta T_{\mathscr{H}}(\delta x) \subseteq \varepsilon_{1} T_{\mathscr{H}}\left(\varepsilon_{1} x\right)$. This contradiction establishes the result.

Second step. $X=\mathbb{R}^{n}, Y$ is a Banach space.

Let $\varepsilon^{\prime}>0$ be as in the first step and $\bar{f}_{1}$ be an $\varepsilon^{\prime}$-approximation of $\left.\bar{F}\right|_{\bar{B}_{\mathscr{X}} \times \bar{V}_{\mathscr{A}}}$ such that its range $\bar{f}_{1}\left(\bar{B}_{\mathscr{H}} \times \bar{V}_{\mathscr{K}}\right)$ is contained in a finite-dimensional subspace $Y_{1} \subset Y$.

Let $V_{\mathscr{M}}^{1}=V_{\mathscr{M}} \cap Y_{1}$, then $S_{\bar{B}_{\mathscr{X}}}^{f_{1}} \subset W \cap\left(\bar{B}_{\mathscr{H}} \times \bar{V}_{\mathscr{M}}^{1}\right)=W_{1}$, let $\bar{f}_{2}=\left.\bar{f}_{1}\right|_{\bar{B}_{\mathscr{X}} \times \bar{V}_{\mathscr{K}}^{1}}$, from the additivity and the reduction properties of the topological degree we have that

$$
\begin{aligned}
& \operatorname{Deg}\left(f_{2}(0, \cdot), W_{1}(0), 0\right)=\operatorname{Deg}\left(f_{1}(0, \cdot), W(0), 0\right) \\
& \quad=\operatorname{Deg}(F(0, \cdot), W(0), 0)=\operatorname{Deg}(F(0, \cdot), V, 0) \neq 0 .
\end{aligned}
$$

Then applying the first step to the maps $\bar{f}_{2}$ and $\bar{G}_{2}=\left.\bar{G}\right|_{\bar{B}_{\mathscr{X}} \times \bar{V}^{1}}$ we obtain that the multivalued map $T_{2}: \bar{B}_{\mathscr{H}} \longrightarrow Y_{1}$, given by $T_{2}(x)=G_{2}\left(x, S^{\bar{f}_{2}}(x)\right), G_{2}=$ 
$I-\bar{G}_{2}$, has a zero $x \in B_{\mathscr{H}}$. As $S_{\bar{B}_{\mathscr{Z}}}^{f_{2}} \subset W$ we have that $\operatorname{Gr} T_{2} \subset \delta \mathrm{Gr} T_{\mathscr{H}}$, contradicting the fact that $0 \notin \varepsilon_{1} T_{\mathscr{H}}\left(\varepsilon_{1} x\right)$.

Third step. $X, Y$ are Banach spaces.

Let $\pi: \mathscr{H} \rightarrow \mathscr{H}_{1}=\mathscr{H} \cap X_{1}$ be a Schauder's $\varepsilon$-projection, $X_{1}$ is a finitedimensional subspace of $X$. Let $\bar{G}_{3}: \bar{B}_{\mathscr{H}} \times \bar{V}_{\mathscr{M}} \bar{G}^{\circ} \circ \mathscr{H}_{1}, \bar{G}_{3}=\pi \circ \bar{G} ; G_{3}=$ $I-\left.\bar{G}_{3}\right|_{\bar{B}_{\mathscr{X}} \times \bar{V}_{\mathscr{R}}}$ and $\bar{F}_{3}=\left.\bar{F}\right|_{\bar{B}_{\mathscr{X}} \times \bar{V}_{\mathscr{Z}}}$. It is clear that $\bar{G}_{3}$ is permissible and if $T^{\prime}$ : $\bar{B}_{\mathscr{O}} \longrightarrow X_{1}$ be defined as $T^{\prime}(x)=G_{3}(x, S(x))$ then $T^{\prime}$ is an $\varepsilon$-approximation of $\left.T\right|_{\bar{B}_{x_{1}}}$ and, for $\varepsilon>0$ sufficiently small, it satisfies the (B.U.) condition on $\partial B_{\mathscr{H}_{1}}$. By the second step we have that $T^{\prime}$ has a zero on $B_{\mathscr{H}_{1}}$, contradicting the fact that $0 \notin \varepsilon T(\varepsilon x)$ for $\varepsilon$ small enough.

Theorem 2.2. Let $X, Y$ be Banach spaces, $U \subset X, V \subset Y$ be open sets. Let $\bar{F}: \bar{U} \times \bar{V} \longrightarrow Y$ be a closed multivalued map with convex values, condensing in the second variable with respect to a regular monotone nonsingular measure of noncompactness $\psi_{Y}$. Let $N \subset \bar{U}$ be a nonempty compact convex set such that $N \subset \mathscr{D}^{F}$ and $\operatorname{Deg}(F(x, \cdot), V, 0) \neq 0$ for some (and hence for all) $x \in N$. If $\bar{G}: \bar{U} \times \bar{V} \longrightarrow X$ is a permissible multivalued set such that $\bar{G}(N, \bar{V}) \subset N$ then there exists a solution $(x, y) \in N \times V$ of system (2.1).

Proof. Let $\rho: X \rightarrow N$ be any retraction and consider the multivalued maps $\bar{F}_{1}: X \times \bar{V}$ ○ $Y, \bar{G}_{1}: X \times \bar{V}-\circ X$ defined as $\bar{F}_{1}(x, y)=\bar{F}(\rho(x), y)$, $\bar{G}_{1}(x, y)=\bar{G}(\rho(x), y)$. Let $B(0, r)$ be any ball containing the set $N$. We may now apply Theorem 2.1 to $\bar{F}_{1}, \bar{G}_{1}$ and $B(0, r)$. It is not difficult to see that all the conditions of this theorem are fulfilled except the closedness of the map $\widehat{G}$, but in our situation this condition is unnecessary since $\widehat{G}$ is compact map.

Hence there exists $(x, y) \in X \times Y$ such that $y \in \bar{F}_{1}(x, y), x \in \bar{G}_{1}(x, y)$. Since $x \in N$ we have that

$$
\left\{\begin{array}{l}
y \in \bar{F}(x, y) \\
x \in \bar{G}(x, y)
\end{array}\right.
$$

In the next theorem we will assume that the multivalued map $\bar{G}$ is admissible and the solution map $x \longrightarrow S(x)$ has acyclic values for any $x \in \mathscr{D}^{F}$.

In order to formulate the result we need to introduce the following boundary condition "P".

- Let $M: \bar{B}(0, r) \subset X \multimap X$ be a multivalued map. We say that $M$ satisfies the boundary condition "P" if $x \in \partial B(0, r)$ and $\lambda x \in M x$ implies $\lambda \leq 1$.

Theorem 2.3. Let $X, Y$ be Banach spaces, $U \subset X, V \subset Y$ be open sets. Let $\psi_{X}$ and $\psi_{Y}$ be monotone nonsingular measures of noncompactness in $X$ and $Y$ respectively. Let $\bar{F}: \bar{U} \times \bar{V} \multimap Y$ be a closed multivalued map $\psi_{Y}$-condensing in the second variable and let $\bar{G}: \bar{U} \times \bar{V} \multimap X$ be an admissible multivalued map. Let us suppose that there exists $r>0$ such that $\bar{B}(0, r) \subset \mathscr{D}^{F}$, and for every $x \in \mathscr{D}^{F}$ the set $S(x)=\{y \in Y: y \in \bar{F}(x, y)\}$ is nonempty and acyclic. Let the multivalued map $\bar{T}: \bar{B}(0, r)-\circ X, \bar{T}(x)=\bar{G}(x, S(x))$, be $\psi_{X}$-condensing and satisfying the property "P" on the boundary $\partial B(0, r)$. Then system (2.1) has a solution.

Proof. The multivalued map $S$ is compact valued and u.s.c. Therefore $\bar{T}$ is an admissible multivalued map. Let $\mathscr{H} \subset X$ be an essential fundamental 
set of $\bar{T}$, since the measure of noncompactness $\psi_{X}$ is nonsingular we may assume, without loss of generality, that $0 \in \mathscr{H}$. We want to show that $\bar{T}$ has a fixed point in $\bar{B}_{\mathscr{H}}=\bar{B}(0, r) \cap \mathscr{H}$. Let $\rho: X \rightarrow \mathscr{H}$ be any retraction then $\widetilde{T}: \bar{B}(0, r) \longrightarrow \circ \mathscr{H}$, given by $\widetilde{T}(x)=\rho \circ \bar{T}(x)$, is an admissible, compact multivalued map. Let us show that it satisfies the property "P". Indeed, if $x \in \partial B(0, r) \cap \mathscr{H}$ then $\widetilde{T}(x)=\bar{T}(x)$ and so the assertion follows directly from our assumptions. If $x \in \partial B(0, r)$ and $x \notin \mathscr{H}$ then $\widetilde{T}(x) \subset \mathscr{H}$ and $\lambda x \cap \mathscr{H}=\varnothing$ for all $\lambda \geq 1$ and hence the property "P" is also fulfilled. Using Lemma 2 of [11] we may find a compact, convex set $\mathscr{N} \subset \bar{B}(0, r)$ such that $\overline{\operatorname{co}}(\Pi \circ \widetilde{T}(\mathcal{N}))=\mathscr{N}$, where $\Pi$ is the radial projection of $X$ on $\bar{B}(0, r)$. Since $\Pi \circ \widetilde{T}(x)$ is admissible, there exists a fixed point $x \in \mathscr{N}$, that is $x \in \Pi \circ \widetilde{T}(x)$. Condition "P" implies that $x \in \widetilde{T}(x)$ and hence $x \in \bar{T}(x)$.

Remark 2.1. We can also prove Theorem 2.3 by using the fact that if $x \notin \bar{T}(x)$ for all $x \in \partial B(0, r)$ then the topological degree $\operatorname{Deg}(T, B(0, r), 0)=1$ (see [3 and 12]).

\section{Applications}

In this section we will give two applications of our results. The first application consists in solving the problem of finding an optimal control parameter, $u \in \mathbb{R}^{n}$, in such a way that the corresponding state vector $y$ of a given control system governed by a neutral functional differential equation, starting from a given convex, compact set $C \subset \mathbb{R}^{n}$ reaches at the final time a variable target set $H(y(0), u) \subset \mathbb{R}^{n}$ and the pair $(y, u)$ minimizes a given functional.

In the second application we look for periodic solutions of a prescribed period of a semilinear evolution differential inclusion in a Banach space.

Example 1. In this example we are looking for a control parameter, represented by a vector $u \in \mathbb{R}^{n}$, in such a way that the following problem is solvable.

$$
\left\{\begin{array}{l}
y^{\prime}(t)=f\left(t, y(t), y(\theta(t)), y^{\prime}(\theta(t)), u\right) \text { for all } t \in[0,1], \\
y(0) \in C, \quad y(1) \in H(y(0), u), \\
\int_{0}^{1} f_{0}\left(y(s), y^{\prime}(s), u\right) d s \rightarrow \min ,
\end{array}\right.
$$

where $C$ is a given convex, compact subset of $\mathbb{R}^{n}$, the multivalued map $H$ : $C \times \mathbb{R}^{n}-\circ \mathbb{R}^{n}$ is closed, compact, and acyclic, $\theta \in C([0,1], \mathbb{R})$ satisfies $0 \leq$ $\theta(t) \leq t$ for any $t \in[0,1]$ and $\theta(0)=0, f_{0}: \mathbb{R}^{n} \times \mathbb{R}^{n} \times \mathbb{R}^{n} \rightarrow \mathbb{R}$ is a continuous function.

We assume the following conditions on the dynamics $f:[0,1] \times \mathbb{R}^{n} \times \mathbb{R}^{n} \times$ $\mathbb{R}^{n} \times \mathbb{R}^{n} \rightarrow \mathbb{R}^{n}$.

$\left(f_{1}\right)$ The map $f$ is continuous at any point

$$
\left(t, v_{1}, v_{2}, v_{3}, u\right) \in[0,1] \times \mathbb{R}^{4 n}
$$

$\left(f_{2}\right)$ there exist positive constants $a, b, c, d$ and $k$, with $0 \leq k<1$, such that

$$
\left|f\left(t, v_{1}, v_{2}, v_{3}, u\right)\right| \leq a+b\left|v_{1}\right|+c\left|v_{2}\right|+k\left|v_{3}\right|+d|u|
$$

for any $\left(t, v_{1}, v_{2}, v_{3}, u\right) \in[0,1] \times \mathbb{R}^{4 n}$;

$\left(f_{3}\right) f\left(t, v_{1}, v_{2}, v_{3}, 0\right)=0$ for any $\left(t, v_{1}, v_{2}, v_{3}\right) \in[0,1] \times \mathbb{R}^{3 n}$; 
$\left(f_{4}\right)$ there exists $N>0$ such that

$$
\liminf _{u \rightarrow+\infty} \frac{\left\langle u, f\left(t, v_{1}, v_{2}, v_{3}, u\right)\right\rangle}{|u|^{1+\delta}} \geq N
$$

for some $0<\delta \leq 1$, for any $t \in[0,1]$ and for every $\left(v_{1}, v_{2}, v_{3}\right)$ belonging to any bounded set of $\mathbb{R}^{3 n}$;

$\left(f_{5}\right)\left|f\left(t, v_{1}, v_{2}, v_{3}, u\right)-f\left(t, v_{1}, v_{2}, v_{3}^{\prime}, u\right)\right| \leq k\left|v_{3}-v_{3}^{\prime}\right|$ for any

$$
\left(t, v_{1}, v_{2}, v_{3}, v_{3}^{\prime}, u\right) \in[0,1] \times \mathbb{R}^{5 n}
$$

where the constant $k$ is the same of assumption $\left(f_{2}\right)$.

Under assumptions $\left(f_{1}\right)-\left(f_{2}\right)$ the problem

$$
y^{\prime}(t)=f\left(t, y(t), y(\theta(t)), y^{\prime}(\theta(t)), u\right), y(0) \in C
$$

can be rewritten in the following form $y \in \bar{F}(x, y)$ where $x=u \in X=\mathbb{R}^{n}$, $y \in Y=C^{1}\left([0,1], \mathbb{R}^{n}\right)$ and the operator $\bar{F}: X \times Y \multimap \circ Y$ is defined by

$$
\bar{F}(x, y)=C+\int_{0}^{t} f\left(s, y(s), y(\theta(s)), y^{\prime}(\theta(s)), x\right) d s .
$$

Furthermore, the operator $\bar{G}: X \times Y \multimap \circ Y$ defined by

$$
\bar{G}(x, y)=x+H(y(0), x)-y(1)
$$

is permissible, in fact $\bar{G}$ can be considered as the composition of the following maps

$W: X \times Y-\circ X \times X$ defined by $W(x, y)=(x-y(1), H(y(0), x)) ;$

$W_{1}: X \times X \rightarrow X$ defined by $W_{1}\left(w_{1}, w_{2}\right)=w_{1}-w_{2}$.

Clearly, $W$ is continuous and $W_{1}$ is u.s.c. with compact, acyclic values, and $\bar{G}=W_{1} \circ W$.

The problem of finding $x \in \mathbb{R}^{n}$ for which a corresponding solution of (3.2) satisfies $y(1) \in H(y(0), x)$ is equivalent to solve the system

$$
\left\{\begin{array}{l}
y \in \bar{F}(x, y) \\
x \in \bar{G}(x, y) .
\end{array}\right.
$$

We will show that this system has a solution by using Theorem 2.1. For this, we need some preliminary results that we present in the sequel.

Lemma 3.1. Assume $\left(f_{1}\right)-\left(f_{2}\right)$. Then for any $r>0$ there exists a constant $M(r)>0$ such that any solution $y$ of (3.2), corresponding to some control $x \in B(0, r)$, satisfies $|y|_{Y} \leq M(r)$.

Proof. Let $y \in Y$ be a solution of the Cauchy problem (3.2) corresponding to $x \in B(0, r)$. By $\left(f_{2}\right)$ we have that

$$
\left|y^{\prime}(t)\right| \leq a_{r}+b|y(t)|+c|y(\theta(t))|+k\left|y^{\prime}(\theta(t))\right|
$$

for any $t \in[0,1]$, where $a_{r}=a+d \cdot r$. Following [17] define now a $C^{1}$-function $z:[0,1] \rightarrow \mathbb{R}$ as follows

$$
z^{\prime}(t)=a_{r}+(b+c) z+k z^{\prime}(t)+1, \quad z(0)=1+|C| .
$$

Or equivalently,

$$
z^{\prime}(t)=\frac{1+a_{r}}{1-k}+\frac{b+c}{1-k} z(t), \quad z(0)=1+|C|,
$$


where $|C|=\max \{|c|: c \in C\}$. Equivalently, we have

$$
\left\{\begin{array}{l}
z^{\prime}(t)=\frac{1+a_{r}}{1-k}+\frac{b+c}{1-k} z(t), \\
z(0)=1+|C| .
\end{array}\right.
$$

It is easy to see that $z$ is a positive and increasing function on $[0,1]$, hence $z^{\prime}(t)$ is also increasing on $[0,1]$ and so

$$
z^{\prime}(t)>a_{r}+b z(t)+c z(\theta(t))+k z^{\prime}(\theta(t))
$$

for any $t \in[0,1]$. We want to show that (3.3) and (3.4) imply $|y|_{Y} \leq|z|_{Y}$. For this, let

$$
\tau=\sup \left\{t \in[0,1]:|y(s)|<z(s) \text { and }\left|y^{\prime}(s)\right|<z^{\prime}(s), 0 \leq s<t\right\} .
$$

Since $|y(0)|<z(0)$ and $\theta(0)=0$, from (3.3) and (3.4) we obtain $\left|y^{\prime}(0)\right|<$ $z^{\prime}(0)$. Therefore $\tau>0$, being $y, z \in Y$. We claim that $\tau=1$. In fact, assume to the contrary that $|y(\tau)|=z(\tau), \tau<1$. Integrating (3.4) between 0 and $\tau$ and using (3.3), the definition of $\tau$ and $0 \leq \theta(t) \leq t$ for any $t \in[0,1]$ we get

$$
z(\tau)-z(0)>\int_{0}^{\tau}\left|y^{\prime}(s)\right| d s .
$$

But $\frac{d}{d t}|y(t)| \leq\left|y^{\prime}(t)\right|$, thus

$$
z(\tau)>z(0)+|y(\tau)|-|y(0)|=1+|y(\tau)| .
$$

Furthermore, if $z(\tau)>|y(\tau)|$ we have, again by (3.3) and (3.4), that $z^{\prime}(\tau)>$ $\left|y^{\prime}(\tau)\right|$. In conclusion, $|y|_{Y}<|z|_{Y}$ and taking $M(r)=|z|_{Y}$ we end the proof.

As a direct consequence of this lemma we have that $\mathscr{D}^{F}=\mathbb{R}^{n}$. Indeed, the map

$$
x \longrightarrow S(x)=\{y \in Y: y \text { is a solution of (3.2) }\}
$$

sends bounded sets into bounded sets, thus, for any $r>0$, we can take $U=$ $B(0, r) \subset X$ and $V=B(0, M(r)) \subset Y$ as open sets in Theorem 2.1.

Definition 3.1. Let $\Omega \subset Y$ be a bounded set, we define a measure of noncompactness $\psi_{Y}$ in $Y$ in the following way $\psi_{Y}(\Omega)=\alpha_{c}\left(\Omega^{\prime}\right)$ where $\Omega^{\prime}=\left\{y^{\prime}\right.$ : $y \in \Omega\}$ and $\alpha_{c}$ is the Kuratowski measure of noncompactness in the space $C\left([0,1], \mathbb{R}^{n}\right)$.

We can now prove the following.

Lemma 3.2. Assume that conditions $\left(f_{1}\right)-\left(f_{2}\right)$ and $\left(f_{5}\right)$ are satisfied. Then the operator $\bar{F}: X \times Y$ - $Y$, given by

$$
\bar{F}(x, y)=C+\int_{0}^{t} f\left(s, y(s), y(\theta(s)), y^{\prime}(\theta(s)), x\right) d s
$$

is $\left(k, \psi_{Y}\right)$-condensing with respect to the second variable.

Proof. Let $K \subset X$ be a compact set, $\Omega \subset Y$ be a bounded set. We want to show that

$$
\psi_{Y}(\bar{F}(K, \Omega)) \leq k \psi_{Y}(\Omega) .
$$


For this, let the operators $h: X \times Y \rightarrow C\left([0,1], \mathbb{R}^{n}\right)$ and

$$
q: X \times C\left([0,1], \mathbb{R}^{n}\right) \times C\left([0,1], \mathbb{R}^{n}\right) \rightarrow C\left([0,1], \mathbb{R}^{n}\right)
$$

be defined as follows

$$
h(x, y)(t)=f\left(t, y(t), y(\theta(t)), y^{\prime}(\theta(t)), x\right)
$$

and

$$
q(x, u, v)(t)=f(t, u(t), u(\theta(t)), v(\theta(t)), x) .
$$

Since $h(K, \Omega) \subset q\left(K, \Omega, \Omega^{\prime}\right)$ it suffices to estimate $\alpha\left(q\left(K, \Omega, \Omega^{\prime}\right)\right)$. The set $T \subset \mathbb{R}^{n}, T=\left\{z: z=y(t)\right.$ or $\left.z=y^{\prime}(t), y \in \Omega, t \in[0,1]\right\}$ is bounded. So, if $\varepsilon>0$ from the uniform continuity of $f$ on $[0,1] \times T^{3} \times K$ it follows that there exists such $\eta>0$ that

$$
\left|f\left(t, u_{1}, u_{2}, v, x\right)-f\left(t, \tilde{u}_{1}, \tilde{u}_{2}, v, \tilde{x}\right)\right|<\varepsilon
$$

whenever $\left|u_{1}-\tilde{u}_{1}\right|+\left|u_{2}-\tilde{u}_{2}\right|+|x-\tilde{x}|<2 \eta, t \in[0,1] ; u_{1}, \tilde{u}_{1}, u_{2}, \tilde{u}_{2}, v \in T$; $x, \tilde{x} \in K$.

As $\Omega$ is a bounded equicontinuous subset of $C\left([0,1] ; \mathbb{R}^{n}\right)$ there is an $\eta$-net $\left\{u_{1}, \ldots, u_{m}\right\}$ for $\Omega$. Let $\left\{k_{1}, \ldots, k_{l}\right\}$ be an $\eta$-net of $K$. Then

$$
q\left(K, \Omega, \Omega^{\prime}\right) \subset \bigcup_{\substack{1 \leq i \leq m, 1 \leq j \leq l}} \varepsilon\left(q\left(k_{j}, u_{i}, \Omega^{\prime}\right)\right) .
$$

From the properties of the Kuratowski measure of noncompactness (see [16]) then it follows that

$$
\alpha\left(q\left(K, \Omega, \Omega^{\prime}\right)\right) \leq \max \left\{\alpha\left(q\left(k_{j}, u_{i}, \Omega^{\prime}\right)+2 \varepsilon: 1 \leq i \leq m, 1 \leq j \leq l\right\} .\right.
$$

Now let us show that the operator $q\left(k_{j}, u_{i}, \cdot\right)$ is $k$-nonexpansive. In fact if $v_{1}, v_{2} \in C\left([0,1] ; \mathbb{R}^{n}\right)$ then

$$
\begin{aligned}
& \left|q\left(k_{j}, u_{i}, v_{1}\right)-q\left(k_{j}, u_{i}, v_{2}\right)\right| \\
& \quad=\sup _{t \in[0,1]}\left|f\left(t, u_{i}(t), u_{i}(\theta(t)), v_{1}(t), k_{j}\right)-f\left(t, u_{i}(t), u_{i}(\theta(t)), v_{2}(t), k_{j}\right)\right| \\
& \quad \leq k \sup _{t \in[0,1]}\left|v_{1}(t)-v_{2}(t)\right|=k\left\|v_{1}-v_{2}\right\| .
\end{aligned}
$$

Therefore

$$
\alpha\left(q\left(k_{j}, u_{i}, \Omega^{\prime}\right)\right) \leq k \alpha\left(\Omega^{\prime}\right)
$$

and, since $\varepsilon>0$ is arbitrary, it follows that

$$
\alpha\left(q\left(K, \Omega, \Omega^{\prime}\right)\right) \leq k \alpha\left(\Omega^{\prime}\right) .
$$

Hence

$$
\psi_{Y}(\bar{F}(K, \Omega))=\alpha(h(K, \Omega)) \leq \alpha\left(q\left(K, \Omega, \Omega^{\prime}\right)\right) \leq k \alpha\left(\Omega^{\prime}\right)=k \psi_{Y}(\Omega) .
$$

This ends the proof.

Lemma 3.3. Assume $\left(f_{1}\right) \div\left(f_{5}\right)$. Then there exists $r>0$ such that:

(i) the multivalued map $T: X-\circ$ defined as follows

$$
\begin{aligned}
T(x) & =G(x, S(x)) \\
& =\bigcup_{y \in S(x)}\left\{\int_{0}^{1} f\left(s, y(s), y(\theta(s)), y^{\prime}(\theta(s)), x\right) d s+C-H(y(0), x)\right\}
\end{aligned}
$$

satisfies the B.U. condition on $\partial B(0, r)$; 
(ii) $\operatorname{Deg}(F(0, \cdot), V, 0) \neq 0$, where $V=B(0, M(r))$.

Proof. By $\left(f_{4}\right)$ and Lemma 3.1 for $r>0$ sufficiently large we have

$$
\operatorname{Deg}(F(0, \cdot), V, 0)=\operatorname{Deg}(I, V, 0) .
$$

By $\left(f_{4}\right)$ and Lemma 3.1, for $r>0$ sufficiently large we have

$$
\frac{\left\langle u, f\left(t, v_{1}, v_{2}, v_{3}, u\right)\right\rangle}{|u|^{1+\delta}}+\inf _{y \in C-\mathscr{C}} \frac{\langle u, y\rangle}{|u|^{1+\delta}} \geq \frac{N}{2}
$$

where $\mathscr{H}=\overline{H(C, X)}$, for any $t \in[0,1]$, for any $u,|u| \geq r$ and any $\left(v_{1}, v_{2}, v_{3}\right) \in \mathbb{R}^{3 n}$ such that $\left|v_{1}\right|+\left|v_{2}\right|+\left|v_{3}\right| \leq 2 M(r)$. In fact,

$$
\lim _{|u| \rightarrow+\infty} \inf _{y \in C-\mathscr{L}} \frac{\langle u, y\rangle}{|u|^{1+\delta}}=0 \text {. }
$$

Finally, if for any $x \in X$ we define $x^{*} \in X^{*}$ as $x^{*}(\cdot)=\langle x, \cdot\rangle$ then it is easy to see that $T$ satisfies the B.U. condition on $\partial B(0, r)$.

By virtue of $\left(f_{3}\right)$ it is evident that $\operatorname{Deg}(F(0, \cdot), V, 0)=\operatorname{Deg}(I, V, 0)$.

We are now in a position to prove the following

Theorem 3.1. Assume $\left(f_{1}\right) \div\left(f_{5}\right)$, then there exists $u \in X$ such that problem (3.1) has a solution $y \in Y$.

Proof. From the proof of Lemma 3.3 it follows that all the solutions of the system

$$
\left\{\begin{array}{l}
y \in \bar{F}(x, y) \\
x \in \bar{G}(x, y)
\end{array}\right.
$$

are contained in $\bar{U} \times \bar{V}$, where $U=B(0, r), V=(0, M(r))$ and the above lemmas show that for $\bar{F}$ and $\bar{G}$ on $\bar{U} \times \bar{V}$ all the assumptions of Theorem 2.1 are satisfied.

From the conditions of Theorem 2.1 it follows that the set $\{(x, y)\}$ of solutions of system 2.1 is a nonempty, compact subset of $X \times Y$. Hence there exists a pair $(y, u) \in Y \times X$ giving the solution of problem (3.1).

Example 2. Let us consider the periodic problem for an evolution semilinear differential inclusion in a separable Banach space $E$ described as follows

$$
y^{\prime}(t) \in A(t) y(t)+\phi(t, y(t)) \quad y(0)=y(1)
$$

under the following assumptions

(A $\left.\mathrm{A}_{1}\right)\{A(t)\}_{t \in[0,1]}$ is a family of closed linear not necessarily bounded operators in $E$ satisfying the periodicity condition $A(0)=A(1)$;

$\left(\mathrm{A}_{2}\right)\{A(t)\}_{t \in[0,1]}$ generates a strongly continuous evolution operator $U$ : $\Delta \rightarrow \mathscr{L}(E)$, where $\Delta=\{(t, s) \in[0,1] \times[0,1]: 0 \leq s \leq t \leq 1\}$ and $\mathscr{L}(E)$ is the space of bounded linear operators defined in $E$.

It is supposed also that $U$ is continuous with respect to the norm of $\mathscr{L}(E)$ while $s<t$.

$\left(\mathrm{A}_{3}\right)$ The operator $U(t, s)$ satisfies the estimation

$$
L=\sup _{(t, s) \in \Delta}\|U(t, s)\|<1 .
$$


It will be supposed that the multivalued map $\phi:[0,1] \times E-\circ E$ has the following properties.

$\left(\phi_{1}\right) \phi$ has compact, convex values;

$\left(\phi_{2}\right) \phi$ is periodic in the first variable, i.e. $\phi(0, \cdot) \equiv \phi(1, \cdot)$;

$\left(\phi_{3}\right) \phi:[0,1] \times E-\circ E$ is u.s.c.; $E$;

$\left(\phi_{4}\right) \phi([0,1] \times E) \subset M$ where $M$ is a weakly compact, convex subset of

$\left(\phi_{5}\right)$ for almost all $t \in[0,1]$ and every bounded $\Omega \subset E$ the set $\phi(t, \Omega)$ is relatively compact in $E$.

In the sequel we will need some additional properties of the Hausdorff measure of noncompactness $\chi$ in $E$.

Lemma 3.4. If $B \in \mathscr{L}(E)$ then $\chi(B(\Omega)) \leq\|B\| \chi(\Omega)$ for every bounded set $\Omega \subset E$.

Lemma 3.5. If $\Omega \subset C([0,1]$; E) is bounded and equicontinuous then

$$
\varphi(\Omega)=\sup _{t \in[0,1]} \chi(\Omega(t))=\chi_{c}(\Omega)
$$

where $\Omega(t)=\{y(t): y \in \Omega\}$ and $\chi_{c}$ is the Hausdorff measure of noncompactness in $C([0,1] ; E)$.

By $S_{Q}^{1}$ we will denote the set of all Bochner integrable selectors of the multifunction $Q:[0,1]-\circ E$, i.e.

$$
S_{Q}^{1}=\left\{q \in L^{1}([0,1] ; E): q(t) \in Q(t) \text { a.e. in }[0,1]\right\} .
$$

If $S_{Q}^{1} \neq \varnothing$ then the multifunction $Q$ is called integrable and

$$
\int_{\tau} Q(s) d s=\left\{\int_{\tau} q(s) d s: q \in S_{Q}^{1}\right\}
$$

for every measurable set $\tau \subset[0,1]$.

A multifunction $Q:[0,1]-\circ E$ is said to be integrably bounded if there exists a nonnegative $\alpha \in L^{1}([0,1])$ such that

$$
\|Q(t)\| \leq \alpha(t) \text { for a.a. } t \in[0,1] .
$$

Lemma 3.6 (cf. [13]). Let the multifunction $Q:[0,1]-\circ E$ be integrable, integrably bounded and $\chi(Q(t)) \leq \gamma(t)$ for a.a. $t \in[0,1]$ where $\gamma \in L^{1}([0,1])$. Then

$$
\chi\left(\int_{\tau} Q(s) d s\right) \leq \int_{\tau} \gamma(s) d s
$$

for every measurable set $\tau \subset[0,1]$.

For $y \in C([0,1] ; E)$ let $H_{y}:[0,1]-\circ E$ be the multifunction given by $H_{y}(t)=\phi(t, y(t))$. From the conditions $\left(\phi_{1}\right),\left(\phi_{3}\right),\left(\phi_{4}\right)$ it follows that $S_{H_{y}}^{1} \neq \varnothing$ for every $y \in C([0,1] ; E)$ (see, for example, [2]).

We will consider the problem of the existence of mild periodic solutions of the inclusion (3.5), i.e. functions $y \in C([0,1] ; E)$ which satisfy to the equation

$$
y(t)=U(t, 0) y(0)+\int_{0}^{t} U(t, s) f(s) d s
$$

where $f \in S_{H_{y}}^{1}$ and to the periodicity condition $y(0)=y(1)$. 
In order to find mild periodic solutions we will consider the spaces $X=E$, $Y=C([0,1] ; E)$ and maps $\bar{F}: X \times Y \multimap \circ Y$, with

$$
\bar{F}(x, y)=\left\{z: z(t)=U(t, 0) x+\int_{0}^{t} U(t, s) f(s) d s ; f \in S_{H_{y}}^{1}\right\}
$$

and $\bar{G}: X \times Y \rightarrow X$, given by $\bar{G}(x, y)=y(1)$.

Then it is clear that the problem (3.5) is equivalent to the system

$$
\left\{\begin{array}{l}
y \in \bar{F}(x, y), \\
x=\bar{G}(x, y) .
\end{array}\right.
$$

Let us show that the multivalued maps $\bar{F}$ and $\bar{G}$ satisfy all the conditions of Theorem 2.3. In fact, using the methods of $[13,15]$, one can prove the following property.

Lemma 3.7. The multivalued map $\bar{F}$ is closed and for every bounded $\mathscr{U} \subset X$ the set $\bar{F}(\mathscr{U}, Y)$ is bounded and equicontinuous.

Lemma 3.8. For every compact $K \subset X$ and bounded $\Omega \subset Y$ the set $\bar{F}(K, \Omega)$ is compact.

Proof. It is clear that

$$
\bar{F}(K, \Omega)(t) \subset U(t, 0) K+\int_{0}^{t} U(t, s) \phi(s, \Omega(s)) d s .
$$

Then from equicontinuity of $\bar{F}(K, \Omega)$ it follows (Lemma 3.5) that

$$
\chi_{Y}(\bar{F}(K, \Omega)) \leq \sup _{t \in[0,1]} \chi_{E}\left[U(t, 0) K+\int_{0}^{t} U(t, s) \phi(s, \Omega(s)) d s\right] .
$$

From condition $\left(\phi_{5}\right)$ it follows that $\chi_{E}(\phi(t, \Omega(t))=0$ a.e. in $[0,1]$ and hence using Lemmas 3.4 and 3.6 we have that

$$
\chi_{Y}(\bar{F}(K, \Omega)) \leq\|U(t, 0)\| \chi_{E}(K)+\int_{0}^{t}\|U(t, s)\| \chi_{E}(\phi(s, \Omega(s))) d s=0
$$

proving the lemma.

Further from Theorem 2.1 of [13] it follows that for every $x \in X$ the set $S(x)=\{y \in Y: y \in \bar{F}(x, y)\}$ is nonempty and using the methods of [15] it can be shown that $S(x)$ is a $R_{\delta}$-set and, hence, acyclic. From $\left(\phi_{6}\right)$ it follows that there exists $\nu \geq 0$ such that

$$
\|\phi(t, e)\|=\sup \{\|y\|: y \in \phi(t, e)\} \leq \nu
$$

for all $(t, e) \in[0,1] \times E$. Now take $B(0, r) \subset X$ where

$$
r>\frac{\nu L}{1-L} \text {. }
$$

Lemma 3.9. The multivalued map $\bar{T}: \bar{B}(0, r)$ - $X, \bar{T}(x)=\bar{G}(x, S(x))$ is $\chi$-condensing and it satisfies the condition

$$
\bar{T}(\partial B(0, r)) \subset B(0, r) .
$$


Proof. Let $\Sigma \subset X$ be a bounded set, then

$\bar{T}(\Sigma) \subset\left\{U(0,1)(\Sigma)+\int_{0}^{1} U(1, s) f(s) d s: f \in S_{H_{y}}^{1}\right.$,

$y$ is solution of $(3.5), y(0) \in \Sigma\}$.

From the condition $\left(\phi_{5}\right)$ and Lemmas 3.4 and 3.6 it follows that

$$
\chi(\bar{T}(\Sigma)) \leq\|U(1,0)\| \chi(\Sigma)
$$

and hence $\bar{T}$ is $(l, \chi)$-condensing where $l=\|U(1,0)\|<1$. Now let $x \in$ $\partial B(0, r)$ and let $z \in \bar{T}(x)$, then

$$
z=U(1,0) x+\int_{0}^{1} U(1, s) f(s) d s
$$

where $f \in S_{H_{y}}^{1}$. Then

$$
\|z\| \leq\|U(1,0)\|\|x\|+\int_{0}^{1}\|U(1, s)\|\|f(s)\| d s \leq L r+L \nu<r .
$$

Now we have the following

Theorem 3.2. Assume $\left(A_{1}\right) \div\left(A_{3}\right)$ and $\left(\phi_{1}\right) \div\left(\phi_{5}\right)$, then there exists a solution of problem (3.5).

Proof. Apply Theorem 2.3.

Remark 3.1. We would like to point out that the differential inclusion in (3.5) can model control systems of a parabolic type (see e.g. [13]).

\section{ACKNOWLEDGMENT}

We would like to thank Professor J. Pejsachowicz for helpful conversations.

\section{REFERENCES}

1. Yu. G. Borisovich, B. D. Gel'man, A. D. Myskis, and V. V. Obukhovskiī, Topological methods in fixed-point theory of multivalued mappings, Uspekhi Mat. Nauk 35 (1980), 59-126; English transl. in Russian Math. Surveys 35 (1980), 65-143.

2. Introduction to the theory of multivalued mappings, Voronezh Univ. Press, Voronezh, (1986); English transl. in J. Soviet Math. 39 (1987), 2772-2811.

3. __, Multivalued analysis and operator inclusions, Ser. Sov. Probl. Mat. Noveishie Dostizheniya, vol. 29, Itogi Nauki i Tekhniki, 1986, pp. 151-211. (Russian)

4. G. Conti, P. Nistri, and P. Zecca, Systems of set-valued equations in Banach spaces, Delay Differential Equations and Dynamical Systems (Proc. Claremont 1990), (S: Busenberg and M. Martelli, eds.), Lecture Notes in Math., vol. 1475, Springer-Verlag, 1991, pp. 98-109.

5. _ Non convex set-valued systems in Banach spaces, Funkcial. Ekvac. (to appear).

6. G. Conti and J. Pejsachowicz, Fixed points theorems for multivalued weighted maps, Ann. Mat. Pura Appl. 126 (1980), 319-341.

7. Do Hong Tan, On continuity of fixed points of multivalued collectively condensing mappings, Indian J. Pure Appl. Math. 15 (1984), 631-632.

8. L. Gorniewicz, Homological methods in fixed point theory of multivalued mappings, Dissertationes Math. 129 (1976), 1-71. 
9. A. Lasota and Z. Opial, An approximation theorem for multivalued mappings, Podstawy Sterowania 1 (1971), 71-75.

10. I. Massabò, P. Nistri, and J. Pejsachowicz, On the solvability of nonlinear equations in Banach spaces, Fixed Point Theory, (Proc. Sherbrooke, Quebec 1980), (E. Fadell and G. Fournier, eds.), Lecture Notes in Math., vol. 886, Springer-Verlag, 1980, pp. 270-289.

11. V. V. Obukhovskii, Some fixed-point principles for multi-valued condensing operators, Voronezh. Gos. Univ. Trudy Mat. Fak. 4 (1970), 70-79. (Russian)

12. $\ldots$ On the topological degree for a class of non compact multivalued mappings, Funktsional Anal. 23 (1984), 82-93. (Russian)

13. __ On semi-linear functional differential inclusions in a Banach space and control systems of a parabolic type, Avtomatika 3 (1991), 73-81. (Russian)

14. V. V. Obukhovskii and E. V. Gorokhov, On the definition of the rotation of a class of compactly restrictible multivalued vector fields, Voronezh. Gos. Univ. Trudy Mat. Fak. 12 (1974), 45-54. (Russian)

15. N. S. Papageorgiou, On multi-valued evolution equations and differential inclusions in Banach spaces, Comment. Math. Univ. St. Paul. 36 (1987), 21-39.

16. B. N. Sadovskii, Limit-compact and condensing operators, Uspekhi Mat. Nauk 27 (1972), no. 1, 81-146. (Russian)

17. E. U. Tarafdar and H. B. Thompson, On the solvability of nonlinear noncompact operator equations, J. Austral. Math. Soc. Ser. A 43 (1987), 103-126.

(P. Nistri and P. Zecca) Dipartimento di Sistemi e Informatica, Università di Firenze, viA S. MARTA N. 3, 50139 Firenze, ItAly

E-mail address: pnistri@vm.idg.fi.cnr.it

E-mail address: pzecca@vm.idg.fi.cnr.it

(V. V. Obukhovskii) Department of Physics and Mathematics, Voronezh State PedaGOGICAL INSTITUTE, 394611 VORONEZH, RUSSIA

E-mail address: aspoergpi.voronezh.su 